\title{
AUTOMATED PETROL PUMP
}

\section{Ruth Moly Benjamin ${ }^{\mathrm{a}}$, Sahana Kannan ${ }^{\mathrm{a}}$, Greeshma K Gopi ${ }^{\mathrm{a}}$ and Reuben George Benjamin ${ }^{\mathrm{a}}$}

Designed and constructed working model of Automated Petrol Station. Our project's salient features include vehicle user ID verification, ensuring the presence of vehicle prior to operation. Secured password to check credit balance and user friendly for filling the petrol without any cash transactions. Fully programmed and tested successfully with microcontroller. Legible display and keypad matrix for easy handling, accurate and safe operation. This working model can be developed further in large scale for implementation to fulfill the requirements in Oman.

Keywords: Proximity Sensor; ISM band; Automated; Encoder;

\section{Introduction}

Due to the increasing advancements in technology in different sectors, the 21 st century is known as the century of technology. In this present day petrol pumps are operated manually (Shaligram Dr. A. D., 2014). Petroleum Stations have become very careful about distribution of Petrol by replacing it with Automated Petrol Pump. ISM (Industrial, Scientific, and Medical) Radio band frequency,(also known as unlicensed bands) has been used in our project which shows a group of band that are internationally reserved for Radio frequency (RF) Energy utilized for industrial, scientific and medical requirements (Techopedia, 2017). Bluetooth devices, Wifi computer networks, cordless phones, etc. make use of ISM bands. ISM devices utilize the ISM frequencies which are generally open frequency bands. For worldwide operations $2.4 \mathrm{GHz}$ ISM band has been widely used.

\section{Literature Review}

A. Embedded Security System using RFID and GSM (Kulkarni A. M., 2015)

The system consists of dual units; one of the unit is placed at the tanker which usually monitors the petrol level in the tank continuously. The petrol level at the start and current petrol level is displayed on the LCD. RFID assembly reads the authentication code of the petrol station. The amount of petrol poured at particular petrol pump including the petrol pump ID is send to central office through GSM techniques. To keep record of all these things visual basic coding.

But RFID is licensed and is more expensive. When the signals from two or more readers overlap reader collision occurs.

B. Multi-Automized Fuel Pump using GSM Technology (Behera S. K., 2013)

In this technology, the main functions performed can be namely divided into two: Customer part and the database. In the first part the customer sends his personal user id and needed amount of petrol. He gets back a password in his GSM. When he comes to petrol station the customer enters this password.

To be filled in his car the specific amount of petrol will be available. The second part, the database contains the customer's user id, account and password. Microcontroller checks the ID in database and gives back the particular coupon password via GSM module of petrol station to the mobile of customer. User enters this password in the LCD which is present in the pump. If he is an authenticated user then the particular amount of petrol will be available to be filled in his car. Microcontroller provides supply to motor according to the amount of petrol requested and then stop when the filling is over. The next is the sensor part. It has three sensors. Temperature in the petrol tank in monitored by temperature sensor and if it is more than a particular critical temperature it notifies the owner of the petrol pump. For avoiding the traffic at the petrol pump IR sensor is used. When a vehicle arrives it increases the count which shows up on the LCD Screen and the count keeps incrementing unless the owner finishes filling petrol in the vehicle. The level of petrol in the tank is monitored by Level Sensor. If it reaches to level one, it gives a notification message to petrol owner about insufficient amount of petrol in tank until the owner refills the petrol tank.

GSM technology might suffer issues due to GSM range and is vulnerable to hacking.

\section{Methodology}

\section{A. Basic Operation}

The DIP switch carries specific IDs for each vehicle in the user side of the ID sender. Encoder scans these IDs which are in the binary form . Once the vehicle reaches the petrol station, the ID detector detects the ID of the vehicle and it is enabled by ID enable. Within ISM band the ASK transmission is employed. When the proximity sensor confirms the presence of vehicle, user may proceed to enter the password through keypad matrix. To display the price the required quantity is

a. Department of Electronics and communication Engineering, Waljat College of Applied Sciences, Muscat, Oman Correspondence: mbruth17@gmail.com 
entered. Two LCD's are used here. The contents which are not constant are displayed on LCD 1 which is controlled by Arduino 256 Mega. For example: amount of petrol, user password, cost etc. The contents which are constant are displayed on LCD2 which is controlled by Arduino UNO. For example: "Waljat College of Applied Science", "Press Start", "Enter Password" etc. We are making use of prepaid cards for money transactions. The required quantity of petrol starts filling in the vehicle when the nozzle is inserted.

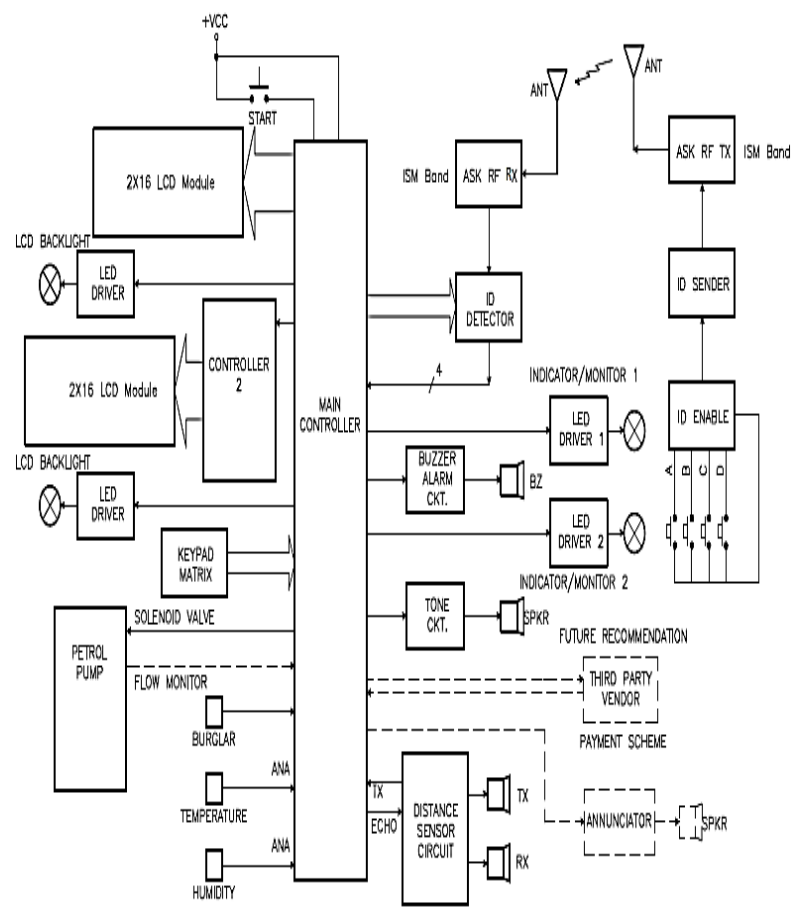

Fig.1 Block Diagram

\section{B. Circuit Operations}

\section{- ID Sender Circuit}

This circuit assigns IDs for a particular vehicle user. The DIP switches presets the ID into 256 unique IDs. In our design, we have limited the number of IDs to four. HT12E encoder scans these IDs which are addressed in binary form. Output is send serially by encoder via Dout. $1 \mathrm{Megohm}$ resistor sets the oscillator frequency as $3 \mathrm{kHz}$.

The addresses A0 to A7 are carried by the push buttons EN-A and EN-D which are the input data. Each of these has a specific task such as to begin the operation of the petrol pump EN-A is pressed.

Under the ISM band of frequency, OOK/ASK wireless transmission is used to send the output of the HT12E encoder.

\section{- ID Enable Circuit}

This circuit is basically works as a dual TOGGLE ON-OFF logic. It works simply by switching between ON and OFF when you press a button, where the LED serves as an indicator.
Four TOGGLE ON-OFF logic are possible for the design out of which only two are used for the project. The output of the ENA is either logic HIGH or LOW which is sent as a data to the ID sender. When a LOGIC HIGH is received the remote devices performs a task otherwise no action at all.

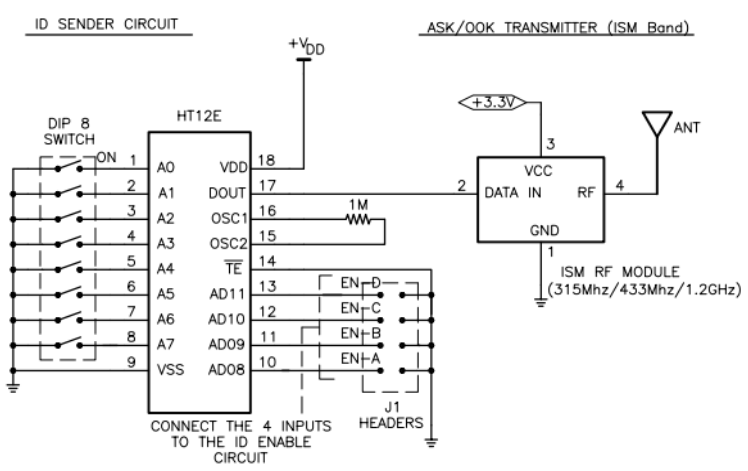

Fig 2.ID Sender Circuit

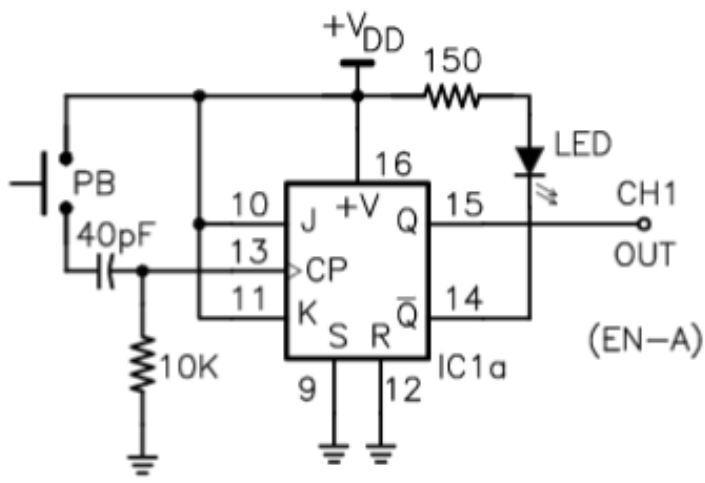

Fig 3. ID Enable Circuit A

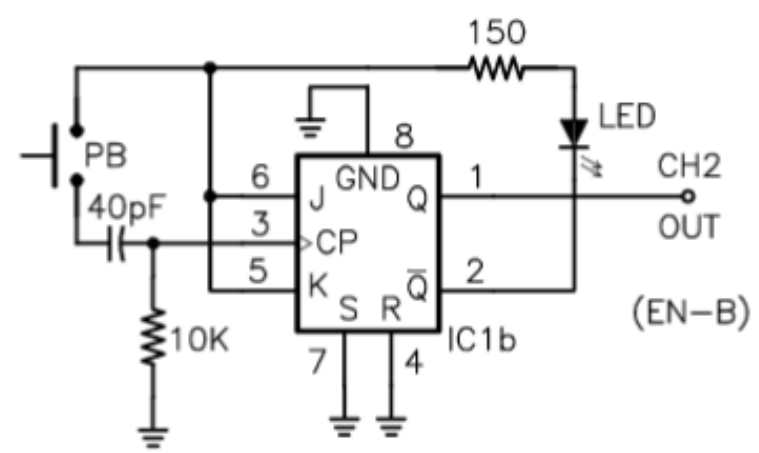

Fig 4.ID Enable Circuit B 


\section{- ID Detector Circuit}

The main section of the petrol station consists of the ID detector circuit. The function of ASK/OOK receiver is to receive signals that resembles its frequency. The selected signal is transformed into binary pulses by the receiver, which is send to DIN of HT12D decoder circuit via the DOUT. The decoder then detects and checks the addresses which are pre adjusted by DIP switches from A0 to A7, with the addresses received from DIN. Transmission is re-directed as an output from A11 to A07 when addresses match.

The microcontroller is introduced in the design as there are numerous vehicle users. The microcontroller presets certain addresses from A0 to A7 up to 256 distinct IDs. The presets of the 256 IDs are continuously sent by the microcontroller and scans to find the addresses that match, which are detected by the HT12D. In this design, the addresses A0 to A1 contains 4 distinct ID's which are sent by the microcontroller. Rest of the ID's are allocated beforehand. Here the system identifies a certain vehicle which matches the IDs and can proceed to the transactions.

Addresses from A08 to A09 denote the output of HT12D and the rest are for future applications. A08 and A09 basically controls the main system and will determine functions like start and initiation of the operation.

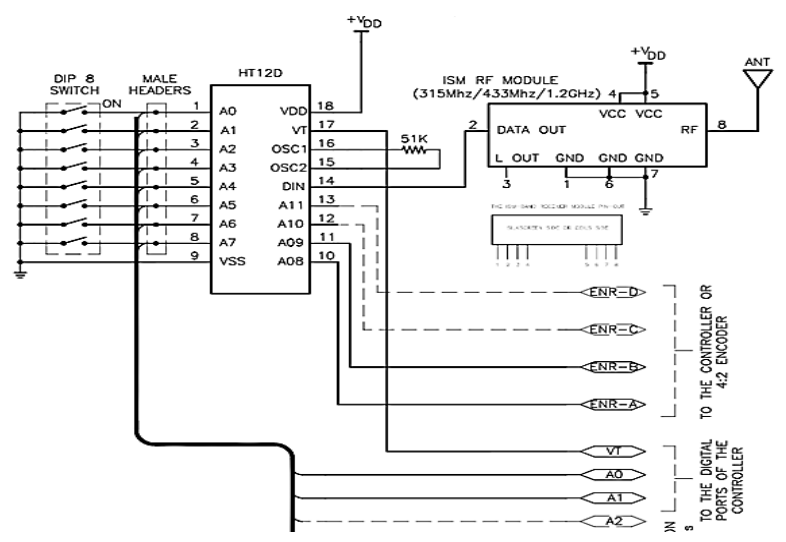

Fig 5.ID Detector Circuit

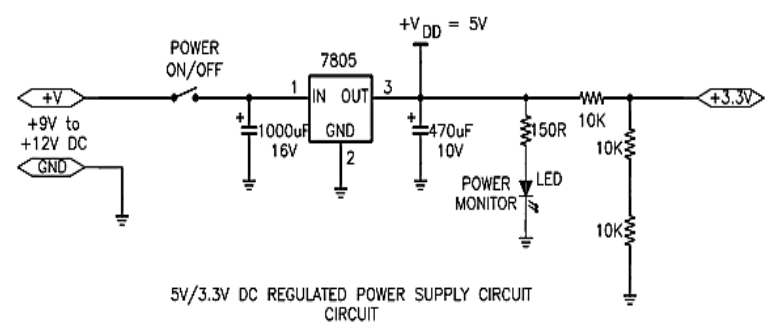

Fig 6.Power Supply to all sections of vehicle user ID circuits
- Main Controller

In the Automated Petrol station the main operation is done by microcontroller. It is assigned to do a sequence of tasks. The microcontroller initiates the output and input operations. The LCD operation is carried out by using General Purpose INPUT/OUTPUT pins. Instructions to the LCD display are send via these pins by the microcontroller. The front end operation of the Automated Petrol Station consists of other pins from P6 and P10 (Sawhney A.K., 2013).

The LCD operation is started by the microcontroller when the power is ON. The driver circuits operation is checked after the LCD operation. The LCD backlight flashes for a few times. The input of the LCD backlight LED driver receives a sequence of logic $\mathrm{HIGH}$ and LOW pulses. The ALARM and STATUS indicators turn ON and OFF the LEDs briefly. MCU alternately transmits logic HIGH and then LOW to the driver inputs for a brief time. Eventually, for a short period of time a buzzing sound is released from the buzzer.

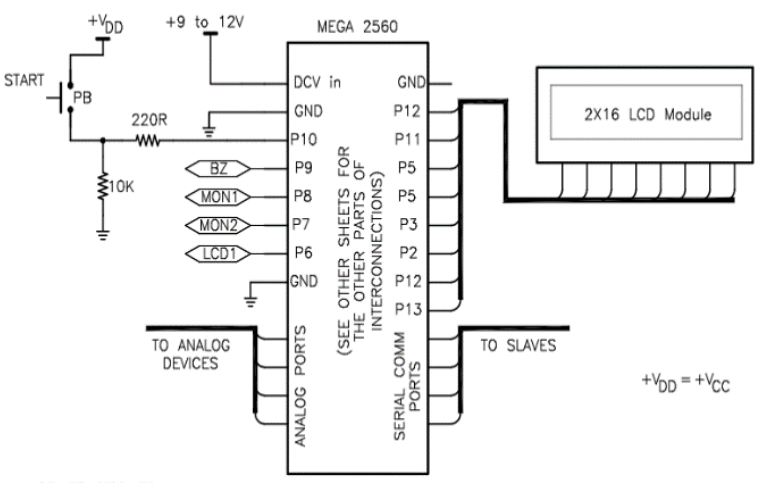

Fig 7. Main Controller Circuit Diagram

The next makes the system wait in the idle state for the START push-button to be pressed ON.

In the idle state, the P10 pin of the MCU receives a LOW logic pulled down by the $10 \mathrm{~K}$ resistor. When the Start button is pressed, MCU receives an input $\mathrm{HIGH}$ from $+5 \mathrm{~V}$ supply. The whole operation of the Automated Petrol Station begins.

\section{Result}

Starting from vehicle entering the station, sequence of operations performed by verification of user ID, vehicle position, password, checking balance, nozzle activation, filling petrol till completing the selected amount or liters and system stopped automatically. Arduino 256Mega and Arduino Uno controls the displays of LCD 1 and LCD 2 respectively and status of every stage of operations displayed, controlled and achieved accurately. 


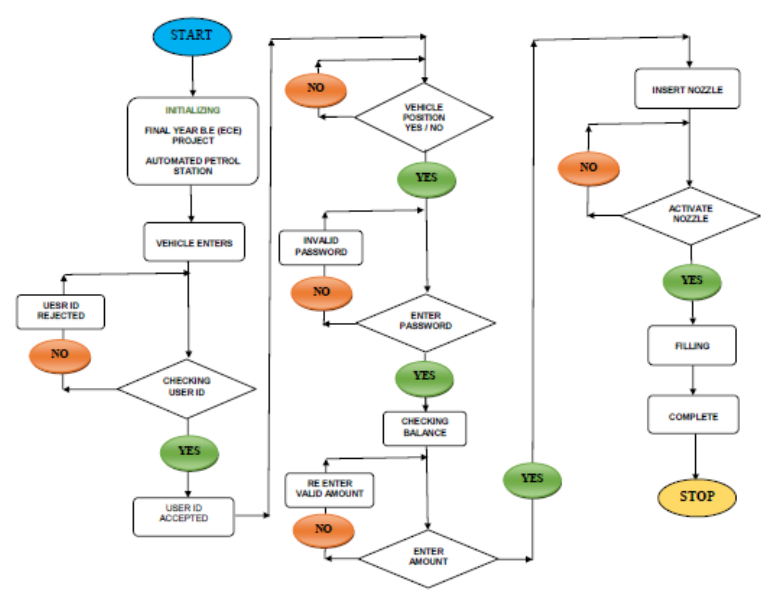

\section{Conclusion}

Fig 8. Flow Chart

We have observed in the recent past that several robbery and murder of petrol station employee in Oman and worldwide. To avoid these incidents, we made an attempt and implemented the automated petrol station for user friendly and safe operation by the consumer directly on cashless transactions.

We have successfully designed and demonstrated the operations of this project by working model.

Automated petrol station will be unmanned operations and reduce or eliminate several risks including:

- No cash transactions

- Minimal time of fueling and no waiting

- Less fueling by error

\section{Acknowledgement}

We would like to express our gratitude to everyone who helped us throughout our project. We are thankful for their support, guidance, and friendly advice during the course of the project work. We would also take this opportunity to thank our Institution and our faculty without whom this project would We also extend our heartfelt thanks to our family and well-wishers.

\section{References}

Kulkarni A. M. and Taware S. S. (2015) Embedded Security System Using RFID \& GSM Modulell (International Journal of Computer Technology \& Electronic Engineering) Volume 2 (Issue 1), Page No. 164-168.

Behera S. K. and Ali Farida A. (2013) Automobile Fuel Pump Control System Using Embedded System\| (International Journal Of Computer Technology \& Electronic Engineering) Volume 3 (Issue 2), Page No. 41-47.

Kapse S. S., Abhale A. A., Kudake C. A., and Shirsath S. B. (2013) Automatic Street Light Control Systeml (International Journal of Emerging Technology and Advanced Engineering) Volume 3, Issue 5.

Techopedia (2017) What Is the Industrial, Scientific and Medical Radio Band (ISM Band)? - Definition from Techopedia". Techopedia.com. n.p.,

Shaligram Dr. A. D., (2014) Sensor and Transducer\| C.T.C, 135.

Sawhney A.K., (2013) Electrical \& Electronic Measurement \& Instrumentationll Dhanpat Rai and Corporation, 993. 